\title{
Value of ultrasound and liver function tests in determining the need for endoscopic retrograde cholangiopancreatography in unexplained abdominal pain
}

\author{
J R Thornton, A J Lobo, D J Lintott, A T R Axon
}

\begin{abstract}
The value of serum liver function tests and abdominal ultrasound as screening tests of the need for endoscopic retrograde cholangiopancreatography (ERCP) was determined in patients with unexplained abdominal pain without associated jaundice. In 1989 and 19901005 ERCPs were undertaken, of which 138 (14\%) were for this indication. The duct or ducts of interest were delineated by ERCP in $95 \%$ of patients. The lesions found were bile duct stones in 10 patients, chronic pancreatitis in five, pancreatic carcinoma in one, peptic ulcer or duodenitis in four. A satisfactory ultrasound examination had been performed in $94 \%$ of patients. For chronic pancreatitis, its sensitivity was $60 \%$ and specificity $95 \%$. For choledocholithiasis, the ultrasonic detection of duct dilatation or stones had a sensitivity of $90 \%$ and specificity of $86 \%$. Of the liver function tests, the alkaline phosphatase was more sensitive $(67 \%)$ than the transaminases $(44 \%)$ in indicating the presence of bile duct stones and had a high specificity (95\%). None of the 10 patients with duct stones had normal ultrasound and normal alkaline phosphatase. Thus it was found that demonstration of a normal common bile duct by abdominal ultrasound and normal serum alkaline phosphatase together have $100 \%$ specificity in excluding bile duct stones. Using such knowledge over the two year period of this study would have spared 36 patients the need for ERCP.

(Gut 1992; 33: 1559-1561)
\end{abstract}

In some patients with chronic abdominal pain, the diagnosis remains uncertain despite extensive investigation. Many of these patients are thought to have a colonic or psychological cause for their pain. ${ }^{1}$ Nevertheless, in those patients in whom the site of the pain includes the epigastrium or right hypochondrium, ERCP is often requested to exclude the possibility of pancreatic or biliary disease which may have been missed by other investigations. ${ }^{1}$

It has been our impression that such patients who were suspected of having pain of biliary origin, but who had had a normal common bile duct demonstrated by abdominal ultrasound and normal serum liver function tests, were never found to have bile duct stones at ERCP. The aim of our study, therefore, was formally to assess this hypothesis.
Methods

\section{PATIENTS}

All the ERCPs carried out at the General Infirmary in 1989 and 1990 were reviewed retrospectively. During this two year period 1005 ERCPs were performed. One hundred and thirty eight $(14 \%)$ were done for unexplained abdominal pain not associated with clinically recognisable jaundice. Four patients had the investigation twice. Therefore, there were 134 patients, of whom the case records were obtained for $130(97 \%)$, enabling anaylsis of 134 ERCPs.

The patients excluded from consideration had well recognised indications for ERCP. These were clinically recognisable jaundice suspected to be obstructive in origin; cholangitis; a definite attack of acute pancreatitis; replacement of a biliary stent; suspected primary sclerosing cholangitis; and the removal of common bile duct stones already visualised at T-tube cholangiography or percutaneous transhepatic cholangiography.

Of the 130 patients with unexplained abdominal pain, $74(57 \%)$ were referred by physicians and $56(43 \%)$ by surgeons. Eighty one (62\%) were patients at either the General Infirmary, or Chapel Allerton Hospital, Leeds. The remaining 49 (38\%) were referred from 10 other hospitals outside the district.

Inspection of the ERCP referral letters and the case notes indicated that the referring consultants considered the patients' pain to be possibly of biliary origin in $75(58 \%)$ and possibly caused by chronic pancreatitis in $30(23 \%)$. In the remaining 25 patients (19\%), it was unclear to us as to the site of the pain suspected by the referring consultants.

The abdominal ultrasound examinations and liver function tests used as the screening tests for ERCP were those performed at the referring hospitals. Some patients had liver function tests measured more than once. If one of the parameters was abnormal on one occasion, but normal on another, the abnormal result was used. Determination of the likely source of a raised serum alkaline phosphatase had rarely been performed.

The sensitivity, specificity, and predictive values of each test were calculated as follows: Sensitivity $=$ (number of patients with disease and positive result)/(all patients with disease tested $) \times 100$. Specificity $=($ number of patients without disease and normal result $) /($ all patients
Correspondence to: Dr J R Thornton, Centre for Digestive Diseases, The General Infirmary at Leeds, Accepted for publication 11 May 1992 
without disease tested) $\times 100$. Positive predictive value $=($ number of patients with disease and positive result)/(all patients with a positive result) $=100$. Negative predictive value $=$ (number of patients without disease and normal result)/(all patients with a normal result) $=100$.

\section{Results}

At ERCP, the duct or ducts of interest were delineated in 123 of the 130 patients $(95 \%)$. A pancreatogram was obtained in all of the 30 patients with suspected pancreatic pain. Eight of these patients, including four found to have chronic pancreatitis, did not have cholagiography either attempted or achieved. A satisfactory cholangiotgram was achieved in 70 of the 75 patients $(93 \%)$ with suspected biliary pain. In the remaining 25 patients, in whom it was unclear from the referral letter and case notes as to the suspected site of the pain, ERCP failed in one and in another a pancreatogram was successful, but the cholangiogram was not obtained.

The positive findings at ERCP were bile duct stones in 10 patients, chronic pancreatitis in five, peptic ulcer or duodenitis in four, and pancreatic carcinoma in one. Of the 10 patients found to have bile duct stones, nine were suspected clinically of having biliary pain. All 10 had their duct stones removed endoscopically without complications. None of the patients with chronic pancreatitis was thought clinically to have pain of biliary origin. Four had this pathology suspected clinically and one case occurred in the group in whom the source of the pain was not identified from the referral letter and case notes. The patient with a pancreatic carcinoma was thought to have biliary pain and this may have been correct as the common bile duct was dilated at ERCP. Excluding the seven patients who had a failed or incomplete investigation, ERCP was negative in 103 patients $(79 \%)$.

Forty four of the 130 patients ( $34 \%$ ) had had a cholecystectomy. This had been performed a median of 4.5 years earlier (range: four months 24 years). The large majority of these patients (37) were suspected of having biliary pain. Forty of these patients had a successful cholangiogram at ERCP. Bile duct stones were found in four of these patients $(10 \%)$ and in six of the 83 patients $(7 \%)$ who had not had a cholecystectomy and had a successful cholangiogram ( $\chi^{2}$, not significant).

Abdominal ultrasound was performed and was technically satisfactory in 122 patients (94\%). The remaining eight patients all had normal ERCP findings, except for one who had a gastric ulcer. Ultrasound showed chronic pancreatitis in three of the five cases in whom this condition was found at ERCP (sensitivity $60 \%$ ). It indicated a normal pancreatic duct in the patient found at ERCP to have a pancreatic carcinoma and a

TABLE Predictive values of ultrasound and liver function tests in the diagnosis of bile duct stones $(\%)$

\begin{tabular}{lll}
\hline & Positive & Negative \\
\hline Ultrasound & 36 & 99 \\
All liver function tests & 44 & 98 \\
Alkaline phosphatase & 50 & 97 \\
Transanimases & 33 & 95 \\
\hline
\end{tabular}

dilated pancreatic duct. In the remaining 115 patients who had an ultrasound and a successful pancreatogram, ultrasound suggested chronic pancreatitis in six cases that was not confirmed by ERCP (specificity 95\%). The predictive values for ultrasound in diagnosing chronic pancreatitis were: positive $33 \%$, negative $98 \%$.

Ultrasound was abnormal, suggesting bile duct dilatation, duct stones or both, in nine of the 10 patients found to have duct stones at ERCP (sensitivity 90\%). These ultrasound abnormalities, however, were also reported in 16 of the 105 patients who at ERCP had a successful cholangiogram and were not found to have duct stones (specificity $85 \%$ ). The predictive values are shown in the Table. In five of these 16 patients, duct dilatation without duct stones was present at ERCP. Thus in these five patients, only one of whom had had a cholecystectomy, the possibility that a stone had been passed in the interval between ultrasound examination and ERCP cannot be excluded. This is rendered less likely by our finding, however, that all five had normal liver function tests.

Liver function tests were performed in 125 of the 130 patients $(96 \%)$, including all the patients with pancreatic disease and nine of the ten patients with duct stones. All these tests were normal in the five patients with chronic pancreatitis. Their predictive values in the diagnosis of choledocholithiasis are shown in the Table. One or more of the liver function tests were abnormal in seven of nine patients with duct stones (sensitivity 78\%) and nine of 111 patients without duct stones and a successful cholangiogram at ERCP (specificity $92 \%$ ). Of the individual tests, the alkaline phosphatase was the most useful, being increased a median of $2 \cdot 3$ times the upper limit of normal (range 1-2-6.4) in six of these nine patients with duct stones (sensitivity 67\%). It was raised in only six of the 111 patients in whom it was measured and who did not have choledocholithiasis found at ERCP (specificity 95\%). The serum transaminases were raised in only four of the nine patients with bile duct stones (sensitivity 44\%) and in eight of the remaining 111 patients who were not found to have stones (specificity 93\%). The serum bilirubin was slightly raised in two patients found to have choledocholithiasis but as mentioned earlier patients with overt jaundice were excluded from this study. Two patients without duct stones also had a minor increase in their serum bilirubin.

None of the 10 patients found to have bile duct stones had a normal common bile duct shown by abdominal ultrasound and a normal serum alkaline phosphatase. With this in mind, it is noteworthy that all seven patients in whom a cholangiogram failed to be achieved at ERCP, had normal liver function tests and abdominal ultrasound.

\section{Discussion}

Endoscopic retrograde cholangiopancreatography is a moderately invasive and expensive investigation and it is therefore particularly desirable that it is used appropriately. The 138 ERCPs in 134 patients with unexplained abdominal pain without accompanying jaundice, 
provided $14 \%$ of our ERCP workload over a two year period. This is a similar proportion to the $17 \%$ reported by others for this type of patient.

In only 20 of our patients (15\%) was ERCP abnormal. This low positivity rate would be even lower if the four patients in whom peptic ulceration or duodenitis were found are excluded on the grounds that their pathology could have been discovered more simply by an upper gastrointestinal endoscopy. We would not wish to imply that the remaining $85 \%$ of these patients had a valueless procedure, as we accept that a negative test is reassuring. It would be preferable, however, if simpler investigations could provide the same degree of reassurance as a negative ERCP.

This may not be possible where chronic pancreatitis is suspected clinically, as we found that abdominal ultrasound was of limited sensitivity $(60 \%)$ and positive predictive value $(33 \%)$ in the detection of this disease. Similarly, computed tomography of the pancreas is also inferior to ERCP in diagnosing this condition. ${ }^{2}$ The liver function tests were unhelpful in these patients, being normal in every case. Thus, even in the absence of an abnormal screening test, we would regard the clinical suspicion of chronic pancreatitis as a reasonable indication for ERCP in such patients.

The majority of our patients with unexplained abdominal pain were suspected clinically of having bile duct stones, but this suspicion was rarely substantiated by ERCP. One third of the total patients had had a cholecystectomy, but this was of little value in predicting the presence of bile duct stones. Thus, duct stones were found at ERCP in only $10 \%$ of those who had had a cholecystectomy compared with $7 \%$ who had not.

Abdominal ultrasound had a low positive predictive value $(36 \%)$ in these patients in indicating choledocholithiasis, usually by showing duct dilatation and occasionally by actually visualising the stones, but had a very high negative predictive value (99\%). As these ultrasound examinations were performed at 12 hospitals, their findings are probably representative of those currently achieved nationally, although they are slightly better than some previous reported results, ${ }^{3-5}$ and similar to one other study. Unfortunately, while abdominal ultrasound alone remains not completely reliable in excluding bile duct stones, some patients who repeatedly complain of epigastric or right upper quadrant abdominal pain will inevitably be referred for the more definitive diagnostic test of ERCP.

Similarly, while an increased alkaline phosphatase with or without a raised transaminase was a useful pointer to the presence of bile duct stones, both these serum markers were normal when measured in two of the nine patients with this problem. As would be expected, the alkaline phosphatase proved to be the more accurate of the two tests in detecting biliary obstruction. It is worth noting, however, that the increase in the alkaline phosphatase in this situation was sometimes very modest.

Thus neither the demonstration of a normal common bile duct by abdominal ultrasound nor liver function tests alone proved entirely satisfactory in ruling out the possibility of bile duct stones as a cause of the patients' pain. When these tests were taken in combination, however, they were $100 \%$ specific in excluding choledocholithiasis. Clearly this finding requires confirmation, particularly as the number of patients found to have duct stones was small.

Over the two year period of the study, if we had not performed ERCP on those patients who were suspected of having biliary pain but who had normal ultrasound and liver function tests, we would have needed to do 36 fewer ERCPs. The British Society of Gastroenterology recently suggested that the cost of a diagnostic ERCP was $£ 118$ plus the radiograph costs. We have estimated the latter to be $£ 42$. Additional costs involved are an overnight hospital stay (£150), preprocedure full blood count, prothrombin time and cross matching of two units of blood (£26), and, for about half the patients, a two-man ambulance journey (estimate £60) with accompanying nurse (estimate £25). Thus the average cost of one of these ERCPs was approximately $£ 379$ and the estimated total cost of all 36 cases, $£ 13,600$. We appreciate, however, that not all of this expenditure is readily saved by not performing these investigations.

Our findings perhaps assume greater importance with the advent of laparoscopic cholectystectomy. The common bile duct is usually not visualised or explored during this procedure. There is consequently uncertainty as to which patients should have preoperative $\mathrm{ERCP}^{8}$ and a few hospitals perform this investigation before laparoscopic cholecystectomy in every patient. On the basis of our data and patients, ERCP is unnecessary for the large majority of such patients providing the patient's abdominal ultrasound shows a normal common bile duct and liver function tests are also normal.

In conclusion, demonstration of a normal common bile duct by abdominal ultrasound and a normal serum alkaline phosphatase together have a $100 \%$ specificity in excluding the presence of bile duct stones. If confirmed by further studies, the use of this information would spare some patients the need for the invasive investigation of ERCP and permit a financial saving.

This study was presented in abstract form to the British Society of Gastroenterology meeting in Manchester, April, 1991.

1 Bull J, Keeling PWN, Thompson RPH. Endoscopic retrograde cholangiopancreatography for unexplained abdominal pain. $B M \mathcal{F} 1980 ; 280: 764$

2 Foster PN, Mitchell CJ,Robertson DRC, Hamilton I, Irving H Kelleher J, et al. Prospective comparison of three noninvasive tests for pancreatic disease. $B M \mathcal{F} 1984 ; 289$ : 13-6.

3 Laing FC, Brooke-Jeffrey R, Wing VW, Nyberg DA. Biliary dilatation: defining the level and cause by real-time ultrasound. Radiology 1986; 160: 39-42.

4 Gibson RN, Yeung E, Thompson JN, Carr DH, Hemingway AP, Bradpiece HA, et al. Bile duct obstruction: radiologic AP, Bradpiece HA, et al. Bile duct obstruction: radiologic 1986; 160: 43-7.

5 Lindsell DRM. Ultrasound imaging of pancreas and biliary tract. Lancet 1990; 335: 390-3.

6 Tobin MV, Mendelson RM, Lamb GH, Gilmore IT. Ultrasound diagnosis of bile duct calculi. $B M \mathcal{F} 1986 ; 293$; 16-7.

7 Wastell C. Laparoscopic cholecystectomy. BMF 1991: 302 $303-4$

8 Thompson JN. Laparoscopic cholecystectomy. (Letter). BMF 1991; 302: 533 . 